\title{
利奈唑胺注射液中主要降解产物的合成
}

\author{
黄志雄 $^{a}$ 桑志培 ${ }^{a} \quad$ 曹海彦 $^{a}$ 晁若冰 ${ }^{a}$ 邓 勇 $*, a, b$ \\ $\left({ }^{a}\right.$ 四川大学华西药学院 成都 610041) \\ ( ${ }^{b}$ 靶向药物及释药系统教育部重点实验室 成都 610041)
}

\begin{abstract}
摘要 以利奈唑胺为起始原料, 利用 $\mathrm{LiOH}$ 选择性水解利奈唑胺的酯键和酰胺键, 得利奈唑胺注射液中的部分水解产 物 $N$-[(2R)-3-[[3-氟-4-(4-吗啉基)苯基]氨基]-2-羟基丙基]-乙酰胺(2)和完全水解杂质(2S)-1-氨基-3-[[3-氟-4-(4-吗啉基)苯 基]氨基]-2-丙醇(4), 收率分别为 78.3\%和 86.6\%; 然后将化合物 $\mathbf{4}$ 的末端氨基用叔丁氧羰基保护, 经 $N, O$-双乙酰化后用 氨水/甲醇脱除 $O$-乙酰基, 再利用饱和氯化氢乙梄溶液脱除叔丁氧羰基保护基, 得 $N$-[(2S)-3-氨基-2-羊基丙基] $-N$-[3-氟 -4-(4-吗啉基)苯基]-乙酰胺(3)盐酸盐, 五步总收率 $62.3 \%$.
\end{abstract}

关键词 噁唑烷酮类抗菌药; 利奈夾胺; 注射液; 降解产物; 杂质; 合成

\section{Synthesis of Major Degradation Products of the Injection of Linezolid}

\author{
Huang, Zhixiong ${ }^{a} \quad$ Sang, Zhipei ${ }^{a} \quad$ Cao, Haiyan $^{a} \quad$ Chao, Ruobing $^{a} \quad$ Deng, Yong*,a,b \\ ( ${ }^{a}$ Department of Medicinal Chemistry, West China School of Pharmacy, Sichuan University, Chengdu 610041) \\ ( ${ }^{b}$ Key Laboratory of Drug Targeting and Drug Delivery System, Chengdu 610041)
}

\begin{abstract}
The ester bond and amide bond of linezolid were selectively hydrolyzed by using LiOH to afford $N-[(2 R)-3-$ [[3-fluoro-4-(4-morpholino)phenyl]amino]-2-hydroxypropyl] acetamide (2) and (2S)-1-amino-3-[[3-fluoro-4-(4-morpholino)phenyl]-amino]-2-propanol (4) with $78.3 \%$ and $86.6 \%$ yields respectively, which were the partially hydrolyzed impurity and fully hydrolyzed impurity of the injection of linezolid. The terminal amino group of compound 4 was then protected with tert-butyloxycarbonyl and subjected to $N, O$-diacetylation. Subsequently, $N$-[(2S)-3-amino-2-hydroxypropyl]- $N$-[3-fluoro-4(4-morpholino)phenyl]acetamide (3) hydrochloride was synthesized by removal of $O$-acetyl with ammonia/methanol and then deprotection tert-butoxycarbonyl using saturated solution of hydrogen chloride in ether with $62.3 \%$ overall yield.

Keywords oxazolidinone antibacterial; linezolid; injection; degradation product; impurity; synthesis
\end{abstract}

利奈唑胺 (linezolid, 1), 化学名为 $(S$ )-5-( 乙酰胺甲 基 )-3-[(3-氟-4-吗啉基) 苯基]-1,3-嚚唑烷-2-酮, 是 Pharmacia \& Upjohn 公司(现 Pfizer 公司)研制的第一个 噁唑烷酮类抗菌药, 2000 年 4 月首次在美国上市, 商品 名 Zyvox®，用于治疗由耐甲氧西林金葡球菌(MRSA)引 起的肺炎和综合性皮肤感染以及由耐万古霉素肠球菌 (VREF)或耐青霉素肺炎链球菌(PRSP)引起的菌血症

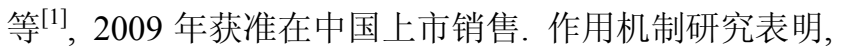
本品可与细菌 $50 \mathrm{~S}$ 亚基附近界面的 $30 \mathrm{~S}$ 亚基结合, 通过 阻止 $70 \mathrm{~S}$ 初始复合物的形成而发挥杀菌作用, 由于作用 于细菌蛋白质合成的早期阶段, 因此本品与其它抗菌药
无交叉耐药性，在体外也不易诱导细菌耐药性的产生 ${ }^{[2]}$, 具有较好的市场开发前景.

在对利奈唑胺注射剂的研制中我们发现，该注射液 在高温灭菌和长期放置过程中极易产生降解杂质，这些 杂质的产生导致利奈唑胺注射液质量降低, 从而影响该 药物临床使用的安全性和有效性, 为此, 需对利奈唑胺 注射液进行质量控制, 而质控过程中需要采用高纯度杂 质作为有关物质对照品或标准品. 目前, 文献报道的利 奈唑胺注射液中的降解杂质主要有以下 4 种 ${ }^{[3]}$, 即: $N$-[(2R)-3-[[3-氟-4-(4-吗啉基)苯基]氨基]-2-羟基丙基]乙酰胺(2)、 $N$-[(2S)-3-氨基-2-羊基丙基]- $N$-[3-氟-4-(4-吗

\footnotetext{
*E-mail: dengyongy@sohu.com

Received December 25, 2013; revised January 16, 2014; published online February 10, 2014.

Project supported by the Research Fund for the Doctoral Program of Higher Education (No. 20110181110079) and the National Science and Technology Major Project (Nos. 2013ZX09301304-002, 2013ZX09202002).

教育部博士点基金(No. 20110181110079)和国家科技重大专项(Nos. 2013ZX09301304-002, 2013ZX09202002)资助项目.
} 
啉基)苯基]-乙酰胺(3)、(2S)-1-氨基-3-[[3-氟-4-(4-吗啉 基)苯基]氨基]-2-丙醇(4)和(5S)-5-(氨甲基)-3-[3-氟-4-(4吗啉基)苯基]-2-噁唑烷酮(5), 其降解途径和降解产物的 化学结构如 Scheme 1 所示. 其中, 杂质 $\mathbf{5}$ 为制备利奈唑 胺原料药的关键中间体, 其合成方法有大量文献报 道 ${ }^{[4]}$, 杂质 2 的合成也有文献报道, 但步骤多, 收率 低 ${ }^{[7,8]}$. 对于杂质 3 和 $\mathbf{4}$, 文献报道采用半制备 HPLC 从 利奈唑胺注射液中用分离得到 ${ }^{[3]}$, 由于利奈唑胺注射液 中杂质众多, 各杂质之间的保留时间非常接近, 通过层 析方式获得高纯度且较大量的单一杂质非常困难, 杂质 3 纯度仅为 $91 \%$, 不能满足作为有关物质对照品或标准
品的要求. 为此, 本文将报道一种反应条件温和、操作 简便、可大量制备较高纯度杂质 $\mathbf{2 , 3}$ 和 $\mathbf{4}$ 的合成方法.

\section{1 结果与讨论}

以利奈唑胺 (1) 为起始原料, 在碱性条件下通过控 制水解条件, 可获得部分水解产物 2 ; 在过量碱和较剧 烈反应条件下, 将利奈唑胺结构中的酯键和酰胺键完全 水解，即可得到 $\mathbf{4}$; 以化合物 4 为原料，通过叔丁氧羰基 (Boc)保护末端胺基后乙酰化，再脱除Boc 保护基，即可 得到 3, 其合成路线如 Scheme 2 所示.

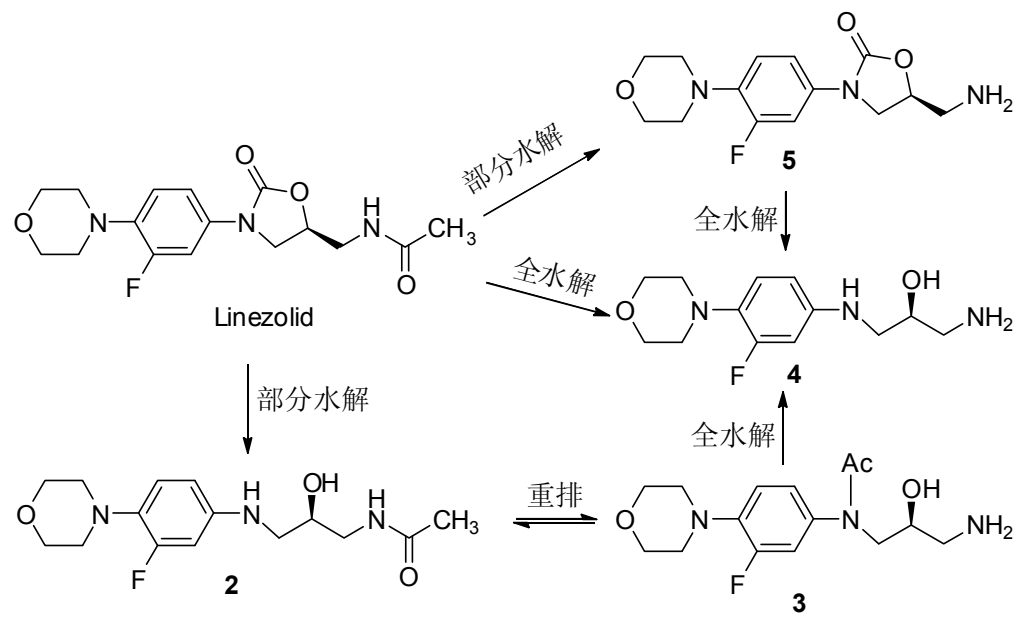

Scheme 1
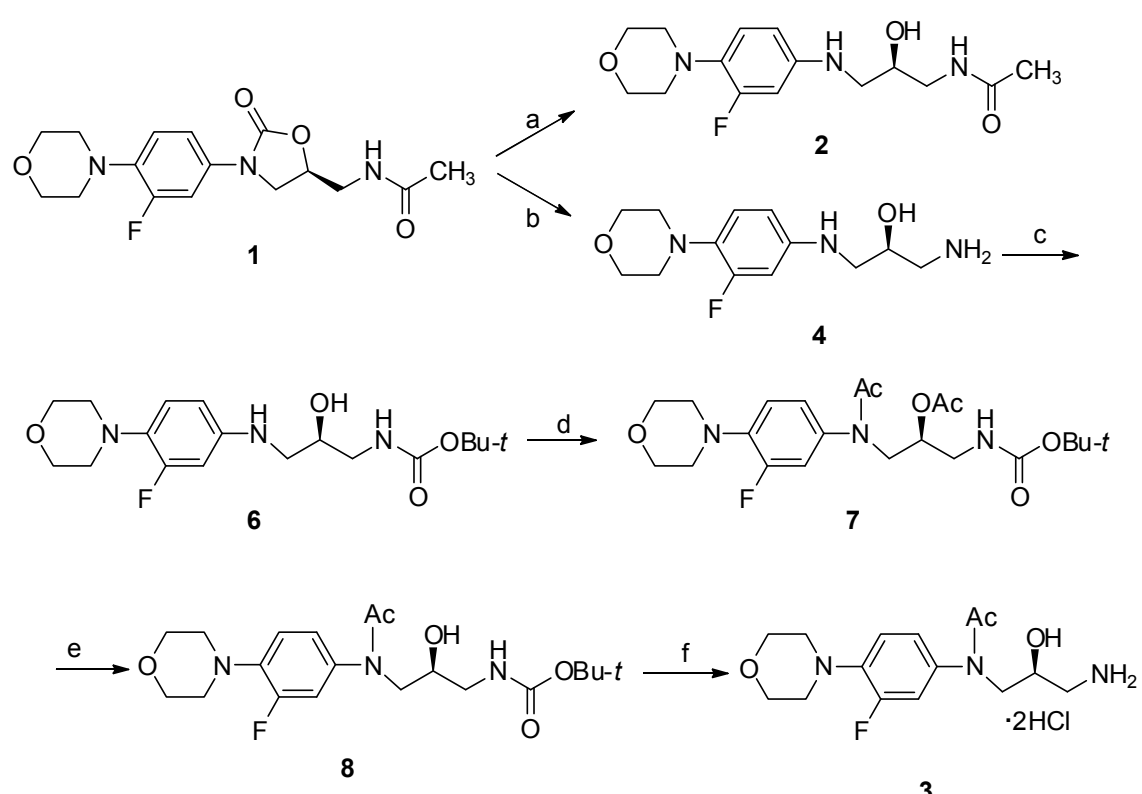

Reagents and conditions: (a) 2.0 equiv. $\mathrm{LiOH}, \mathrm{MeOH} / \mathrm{H}_{2} \mathrm{O}$, reflux, $2 \mathrm{~h}, 78.3 \%$; (b) 5.0 equiv. $\mathrm{LiOH}, \mathrm{MeOH} / \mathrm{H}_{2} \mathrm{O}$, reflux, $8 \mathrm{~h}, 86.6 \%$; (c) $\mathrm{Boc} 2 \mathrm{O}$, $\mathrm{NaHCO}_{3}, \mathrm{MeOH} / \mathrm{H}_{2} \mathrm{O}$, r.t., 2 h, $96.0 \%$; (d) 2.2 equiv. $\mathrm{CH}_{3} \mathrm{COCl}$, $\mathrm{Et}_{3} \mathrm{~N}, \mathrm{CH}_{2} \mathrm{Cl}_{2}$, r.t., 3 h, $95.0 \%$; (e) $\mathrm{NH}_{3} / \mathrm{H}_{2} \mathrm{O}$, $\mathrm{MeOH}$, r.t., 12 h, $85.0 \%$; (f) $\mathrm{HCl} / \mathrm{Et}_{2} \mathrm{O}, 0{ }^{\circ} \mathrm{C}, 2 \mathrm{~h}, 92.7 \%$.

\section{Scheme 2}


由于利奈唑胺分子结构中存在酯键和酰胺键, 用 $\mathrm{LiOH}$ 水解时可通过控制其用量和反应时间达到选择性 水解酯键的目的, 研究发现, 采用 1.2 和 1.5 equiv.的 $\mathrm{LiOH}$ 在甲醇/水混合溶剂回流反应 $2 \mathrm{~h}$, 虽化合物 2 为主 要产物, 但仍有约 $20 \%$ 利奈唑胺不能水解, 继续延长反 应时间, 完全水解产物化合物 4 的量明显增加, 当使用 2.0 equiv.的 $\mathrm{LiOH}$ 回流水解 $2 \mathrm{~h}$, 利奈唑胺基本消失, 且 化合物 4 的量低于 $10 \%$, 经柱层析纯化即可得到纯度为 99.56\%的化合物 2 .

对于化合物 $\mathbf{4}$ 的合成，采用大大过量的的 LiOH (5.0 equiv.)来水解利奈唑胺, 可得到单一产物, 经乙醇重结 晶即可得到纯度为 $99.8 \%$ 的化合物 4 .

对于化合物 3 的合成, 由于乙酰基位于亲核能力低 的苯胺氮原子, 不能将化合物 4 直接乙酰化得到, 为此, 我们先利用 Boc 保护化合物 $\mathbf{4}$ 末端氨基, 所得中间体 $\mathbf{6}$ 再与乙酰氯反应, 可将苯胺氮原子乙酰化, 我们发现, 使用 1.2 和 1.5 equiv. 的乙酰氯反应时, 反应主产物为 $N$ 乙酰化的化合物 8, 但存在一定数量的 $O$-乙酰化和 $N, O$ 双乙酰化副产物, 分离纯化困难, 为此, 我们将乙酰氯 用量增加到 2.2 equiv., 使化合物 6 发生 $N, O$-双乙酰化, 得单一化合物 7 , 不需纯化直接用于下步反应. 所得化 合物 7, 采用常规方法氨解, 脱除 $O$-乙酰基, 得化合物 8 , 再利用饱和氯化氢乙醚溶液脱除 Boc 保护基, 得化 合物 $\mathbf{3}$ 的盐酸盐, 纯度为 $98.6 \%$. 实验发现, 化合物 $\mathbf{3}$ 的 盐酸盐在碱性条件下极易重排为化合物 $\mathbf{2}$, 其反应式如 Eq. 1 所示, 因此, 制备得到的化合物 3 需以盐的形式保 存.

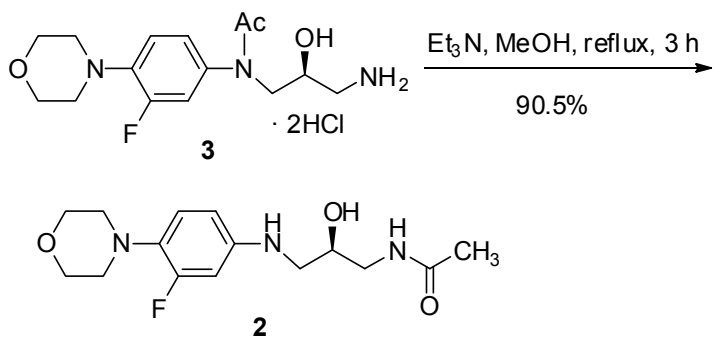

\section{2 结论}

以利奈唑胺(1)为起始原料, 利用 $\mathrm{LiOH}$ 选择性水解 利奈唑胺结构中的酯键和酰胺键, 制得利奈唑胺注射液 中的部分水解杂质 $\mathbf{2}$ 和完全水解杂质 $\mathbf{4}$, 收率分别为 $78.3 \%$ 和 $86.6 \%$; 然后以化合物 4 为原料, 通过 Boc 保护 其末端氨基, 经 $N, O$-双乙酰化后用氨水/甲醇脱除 $O$-乙 酰基, 再利用饱和氯化氢乙醚溶液脱除 Boc 保护基, 得 化合物 3 的盐酸盐, 五步总收率约 $62.3 \%$ (以利奈唑胺 计); 研究发现, 化合物 3 的盐酸盐在碱性条件下极易重 排为化合物 2. 上述各中间体和目标物的化学结构经 ${ }^{1} \mathrm{H}$
NMR, ${ }^{13} \mathrm{C}$ NMR 和 ESI-MS 确证, 其纯度经 HPLC 测定.

\section{3 实验部分}

\section{1 仪器与试剂}

TYR-3 熔点仪, 温度计未经校正; Varian INOVA400 核磁共振仪(溶剂为 $\mathrm{D}_{2} \mathrm{O}, \mathrm{CDCl}_{3}$ 或 DMSO- $d_{6}$, 内标 TMS); Agilent-6210 TOF LC/MS 高分辨质谱仪. 利奈唑 胺原料为工业品(纯度 $99.9 \%, e e$ 为 $99.9 \%$ ), 所用其它试 剂均为市售分析纯或化学纯, 并根据需要进行常规处 理. HPLC 纯度测定条件: 色谱柱为 Gemini C18 (150 $\mathrm{mm} \times 4.6 \mathrm{~mm}, 5 \mu \mathrm{m})$; 流动相为三氟醋酸乙腈溶液 $(10 \%$ 三氟醋酸 $10 \mathrm{~mL}$ 加入 $1000 \mathrm{~mL}$ 乙腈中); 流速为 1.0 $\mathrm{mL} / \mathrm{min}$; 检测波长为 $254 \mathrm{~nm}$.

\section{$3.2 N-[(2 R)-3$-[[3-氟-4-(4-吗啉基)苯基]氨基]-2-羟基 丙基]-乙酰胺(2)的制备}

将利奈唑胺 $(10.12 \mathrm{~g}, 0.03 \mathrm{~mol}) 、$ 甲醇 $(75 \mathrm{~mL}) 、$ 去离 子水 $(30 \mathrm{~mL})$ 和氢氧化锂 $(2.6 \mathrm{~g}, 0.06 \mathrm{~mol})$ 加入反应瓶中, 升温回流搅拌反应 $2 \mathrm{~h}$, 反应结束后, 减压蒸除溶剂, 残 余物用二氯甲烷 $(75 \mathrm{~mL})$ 溶解, 依次用去离子水 $(30 \mathrm{~mL})$ 及饱和 $\mathrm{NaCl}$ 水溶液 $\left(30 \mathrm{~mL}\right.$ ) 洗涤, 有机层经无水 $\mathrm{Na}_{2} \mathrm{SO}_{4}$ 干燥, 减压蒸除溶剂, 残余物经硅胶柱层析纯化 [洗脱 液: $V$ (二氯甲烷) $: V$ (甲醇 $)=30: 1$ ], 得 $7.3 \mathrm{~g}$ 白色固体, 收率 78.3\%, 纯度 $99.56 \%$ (HPLC 归一化法). m.p. $124.5 \sim 125.7{ }^{\circ} \mathrm{C} ;[\alpha]_{\mathrm{D}}^{20}-0.9\left(c 1.0, \mathrm{CH}_{3} \mathrm{OH}\right) ;{ }^{1} \mathrm{H}$ NMR (DMSO- $\left.d_{6}, 400 \mathrm{MHz}\right) \delta: 7.86$ (t, $J=4.8 \mathrm{~Hz}, 1 \mathrm{H}, \mathrm{NHCO}$ ), $6.82(\mathrm{t}, J=9.6 \mathrm{~Hz}, 1 \mathrm{H}, \mathrm{ArH}), 6.41(\mathrm{dd}, J=2.4,15.2 \mathrm{~Hz}$, $1 \mathrm{H}, \mathrm{ArH}), 6.33$ (dd, $J=2.4,8.4 \mathrm{~Hz}, 1 \mathrm{H}, \mathrm{ArH}), 5.47$ (t, $J=$ $5.6 \mathrm{~Hz}, 1 \mathrm{H}, \mathrm{ArNH}), 4.97(\mathrm{~d}, J=5.2 \mathrm{~Hz}, 1 \mathrm{H}, \mathrm{OH}), 3.69$ (t, $\left.J=4.8 \mathrm{~Hz}, 4 \mathrm{H}, 2 \times \mathrm{CH}_{2} \mathrm{O}\right), 3.65 \sim 3.60(\mathrm{~m}, 1 \mathrm{H}, \mathrm{CHOH})$, $3.18 \sim 3.12\left(\mathrm{~m}, 1 \mathrm{H}, \mathrm{CH}_{2} \mathrm{NHCO}\right), 3.07 \sim 3.00(\mathrm{~m}, 1 \mathrm{H}$, $\mathrm{CH}_{2} \mathrm{NHCO}$ ), $3.00 \sim 2.96\left(\mathrm{~m}, 1 \mathrm{H}, \mathrm{CH}_{2} \mathrm{NHAr}\right.$ ), $2.89 \sim 2.85$ (m, $1 \mathrm{H}, \mathrm{CH}_{2} \mathrm{NHAr}$ ), $2.82\left(\mathrm{t}, J=4.8 \mathrm{~Hz}, 4 \mathrm{H}, 2 \times \mathrm{CH}_{2} \mathrm{~N}\right.$ ), $1.82\left(\mathrm{~s}, 3 \mathrm{H}, \mathrm{COCH}_{3}\right) ;{ }^{13} \mathrm{C}$ NMR (DMSO- $\left.d_{6}, 100 \mathrm{MHz}\right) \delta$ : 169.8, $156.7(\mathrm{~d}, J=240.7 \mathrm{~Hz}), 146.1(\mathrm{~d}, J=10.5 \mathrm{~Hz})$, 129.2 (d, $J=9.8 \mathrm{~Hz}), 120.7$ (d, $J=4.5 \mathrm{~Hz}), 108.0$ (d, $J=$ $2.3 \mathrm{~Hz}$ ), 100.5 (d, $J=24.2 \mathrm{~Hz}$ ), 68.2, 66.6, 51.8, 47.7, 43.4, 22.8; HRMS (ESI) calcd for $\mathrm{C}_{15} \mathrm{H}_{23} \mathrm{FN}_{3} \mathrm{O}_{3}[\mathrm{M}+\mathrm{H}]^{+}$ 312.1723 , found 312.1716 .

\section{3 (2S)-1-氨基-3-[[3-氟-4-(4-吗啉基)苯基]氨基]-2-丙 醇(4)的制备}

将利奈唑胺 $(10.12 \mathrm{~g}, 0.03 \mathrm{~mol}) 、$ 甲醇 $(75 \mathrm{~mL}) 、$ 去离 子水 $(30 \mathrm{~mL})$ 和氢氧化锂 $(6.3 \mathrm{~g}, 0.15 \mathrm{~mol})$ 加入反应瓶中, 升温回流摚拌反应 $8 \mathrm{~h}$, 反应结束后, 减压蒸除溶剂, 残 余物用二氯甲烷 $(75 \mathrm{~mL})$ 溶解, 依次用去离子水 $(30 \mathrm{~mL})$ 
和饱和 $\mathrm{NaCl}$ 水溶液 $(30 \mathrm{~mL})$ 洗涤, 有机层经无水 $\mathrm{Na}_{2} \mathrm{SO}_{4}$ 干燥, 减压蒸除溶剂, 残余物经乙醇重结晶, 得 $7.0 \mathrm{~g}$ 白 色固体, 收率 $86.6 \%$, 纯度 $99.8 \%$ (HPLC归一化法). m.p. $99.5 \sim 100.5{ }^{\circ} \mathrm{C} ;[\alpha]_{\mathrm{D}}^{20}+1.4\left(c 1.0, \mathrm{CH}_{3} \mathrm{OH}\right) ;{ }^{1} \mathrm{H}$ NMR (DMSO- $\left.d_{6}, 400 \mathrm{MHz}\right) \delta: 6.81(\mathrm{t}, J=9.6 \mathrm{~Hz}, 1 \mathrm{H}, \mathrm{ArH})$, 6.41 (dd, $J=2.4,15.2 \mathrm{~Hz}, 1 \mathrm{H}, \mathrm{ArH}), 6.34$ (dd, $J=2.4,8.8$ $\mathrm{Hz}, 1 \mathrm{H}, \mathrm{ArH}), 5.46$ (t, $J=5.2 \mathrm{~Hz}, 1 \mathrm{H}, \mathrm{ArNH}$ ), 4.72 (brs, $1 \mathrm{H}, \mathrm{OH}), 3.69\left(\mathrm{t}, J=4.8 \mathrm{~Hz}, 4 \mathrm{H}, 2 \times \mathrm{CH}_{2} \mathrm{O}\right), 3.51 \sim 3.47$ $(\mathrm{m}, 1 \mathrm{H}, \mathrm{CHOH}), 3.06 \sim 3.00\left(\mathrm{~m}, 1 \mathrm{H}, \mathrm{CH}_{2} \mathrm{NHAr}\right), 2.87 \sim$ $2.82\left(\mathrm{~m}, 1 \mathrm{H}, \mathrm{CH}_{2} \mathrm{NHAr}\right), 2.81(\mathrm{t}, J=4.8 \mathrm{~Hz}, 4 \mathrm{H}$, $2 \times \mathrm{CH}_{2} \mathrm{~N}$ ), 2.59 (dd, $\left.J=4.8,12.8 \mathrm{~Hz}, 1 \mathrm{H}, \mathrm{CH}_{2} \mathrm{NH}_{2}\right), 2.47$ (dd, $J=4.8,12.8 \mathrm{~Hz}, 1 \mathrm{H}, \mathrm{CH}_{2} \mathrm{NH}_{2}$ ), 1.55 (brs, $2 \mathrm{H}, \mathrm{NH}_{2}$ ); ${ }^{13} \mathrm{C}$ NMR (DMSO- $\left.d_{6}, 100 \mathrm{MHz}\right) \delta: 156.7$ (d, $J=240.7$ $\mathrm{Hz}), 146.3$ (d, $J=10.6 \mathrm{~Hz}), 129.0$ (d, $J=9.8 \mathrm{~Hz}), 120.7$, 107.9, 100.4 (d, $J=24.5 \mathrm{~Hz}), 70.7,66.6,51.8,47.7,46.1$; HRMS (ESI) calcd for $\mathrm{C}_{13} \mathrm{H}_{21} \mathrm{FN}_{3} \mathrm{O}_{2}[\mathrm{M}+\mathrm{H}]^{+}$270.1618, found 270.1610 .

\section{4 (2R)- $N$-叔丁氧羰基-1-氨基-3-[[3-氟-4-(4-吗啉基)} 苯基]氨基]-2-丙醇(6)的制备

在反应瓶中依次加入中间体 $4(5.39 \mathrm{~g}, 0.02 \mathrm{~mol})$ 、甲 醇 $(60 \mathrm{~mL})$ 、去离子水 $(30 \mathrm{~mL})$ 和碳酸氢钠 $(2.1 \mathrm{~g}, 0.025$ $\mathrm{mol})$, 搅拌均匀后, 加入 $\mathrm{Boc}_{2} \mathrm{O}(5.46 \mathrm{~g}, 0.025 \mathrm{~mol})$, 室 温摚拌反应 $2.0 \mathrm{~h}$, 反应结束后, 减压蒸除溶剂, 残余物 用二氯甲烷 $(75 \mathrm{~mL})$ 溶解, 有机层依次用去离子水 $(30$ $\mathrm{mL}$ ) 和饱和 $\mathrm{NaCl}$ 水溶液 $(30 \mathrm{~mL})$ 洗涤, 经无水 $\mathrm{Na}_{2} \mathrm{SO}_{4}$ 干 燥后减压蒸除溶剂, 得 $7.1 \mathrm{~g}$ 无色油状物, 收率 $96.0 \%$. $[\alpha]_{\mathrm{D}}^{20}+4.4\left(c 1.0, \mathrm{CH}_{3} \mathrm{OH}\right) ;{ }^{1} \mathrm{H} \mathrm{NMR}\left(\mathrm{CDCl}_{3}, 400 \mathrm{MHz}\right)$ $\delta: 6.85$ (t, $J=8.8 \mathrm{~Hz}, 1 \mathrm{H}, \mathrm{ArH}), 6.45 \sim 6.38(\mathrm{~m}, 2 \mathrm{H}, \mathrm{ArH})$, 4.95 (brs, 1H, NHCO), 3.95 3.91 (m, 1H, CHOH), 3.87 $\left(\mathrm{t}, J=4.4 \mathrm{~Hz}, 4 \mathrm{H}, 2 \times \mathrm{CH}_{2} \mathrm{O}\right), 3.35 \sim 3.24(\mathrm{~m}, 4 \mathrm{H}$, $\mathrm{CH}_{2} \mathrm{NHAr}+\mathrm{CH}_{2} \mathrm{NHCO}$ ), 3.23 (brs, $2 \mathrm{H}, \mathrm{OH}+\mathrm{NH}$ ), 2.99 (t, $J=4.8 \mathrm{~Hz}, 4 \mathrm{H}, 2 \times \mathrm{CH}_{2} \mathrm{~N}$ ), $1.46\left(\mathrm{~s}, 9 \mathrm{H}, \mathrm{CH}_{3}\right)$; HRMS (ESI) calcd for $\mathrm{C}_{18} \mathrm{H}_{29} \mathrm{FN}_{3} \mathrm{O}_{4}[\mathrm{M}+\mathrm{H}]^{+}$370.2142, found 370.2139 .

\section{5 (2S)- $N$-叔丁氧羰基-1-氨基-3-[[3-氟-4-(4-吗啉基) 苯基]乙酰氨基]-2-丙醇乙酸酯(7)的制备}

将中间体 $6(5.54 \mathrm{~g}, 0.015 \mathrm{~mol})$ 、二氯甲烷 $(60 \mathrm{~mL})$ 和三乙胺 $(5.6 \mathrm{~mL}, 0.04 \mathrm{~mol})$ 加入反应瓶中, 摚拌均匀后, 置冰浴中冷却至 $0 \sim 5{ }^{\circ} \mathrm{C}$, 滴加乙酰氯 $(2.4 \mathrm{~mL}, 0.033$ $\mathrm{mol}$ ), 控制反应温度不超过 $10{ }^{\circ} \mathrm{C}$, 滴毕, 室温摚拌反应 $3 \mathrm{~h}$, 反应液依次用 $\mathrm{NaHCO}_{3}$ 水溶液 $(30 \mathrm{~mL}$ ) 和饱和 $\mathrm{NaCl}$ 水溶液 $\left(30 \mathrm{~mL}\right.$ ) 洗涤, 有机层经无水 $\mathrm{Na}_{2} \mathrm{SO}_{4}$ 干燥, 减压 蒸除溶剂, 得 $6.46 \mathrm{~g}$ 淡黄色油状物, 收率 $95.0 \%$. [ $\alpha]_{\mathrm{D}}^{20}$ -3.1 (c 1.0, $\left.\mathrm{CH}_{3} \mathrm{OH}\right) ;{ }^{1} \mathrm{H}$ NMR $\left(\mathrm{CDCl}_{3}, 400 \mathrm{MHz}\right) \delta$ : 6.94 6.91 (m, 3H, ArH), 5.36 (brs, 1H, NHCO), 4.92 $4.89(\mathrm{~m}, 1 \mathrm{H}, \mathrm{CHOH}), 3.95(\mathrm{dd}, J=5.6,14.4 \mathrm{~Hz}, 1 \mathrm{H}$, $\left.\mathrm{CH}_{2} \mathrm{NAr}\right), 3.88\left(\mathrm{t}, J=4.4 \mathrm{~Hz}, 4 \mathrm{H}, 2 \times \mathrm{CH}_{2} \mathrm{O}\right), 3.83(\mathrm{dd}$, $\left.J=5.6,14.4 \mathrm{~Hz}, 1 \mathrm{H}, \mathrm{CH}_{2} \mathrm{NAr}\right), 3.46 \sim 3.41(\mathrm{~m}, 1 \mathrm{H}$, $\mathrm{CH}_{2} \mathrm{NHCO}$ ), $3.27 \sim 3.22\left(\mathrm{~m}, 1 \mathrm{H}, \mathrm{CH}_{2} \mathrm{NHCO}\right), 3.11$ (t, $J=$ $\left.4.8 \mathrm{~Hz}, 4 \mathrm{H}, 2 \times \mathrm{CH}_{2} \mathrm{~N}\right), 1.93$ (s, $\left.3 \mathrm{H}, \mathrm{CH}_{3} \mathrm{CO}\right), 1.87$ (s, $3 \mathrm{H}$, $\mathrm{CH}_{3} \mathrm{CO}$ ), $1.44\left(\mathrm{~s}, 9 \mathrm{H}, \mathrm{CH}_{3}\right.$ ); HRMS (ESI) calcd for $\mathrm{C}_{22} \mathrm{H}_{33} \mathrm{FN}_{3} \mathrm{O}_{6}[\mathrm{M}+\mathrm{H}]^{+}$454.2353, found 454.2358.

\section{6 (2S)- $N$-叔丁氧羰基-1-氨基-3-[[3-氟-4-(4-吗啉基)} 苯基]乙酰氨基]-2-丙醇(8)的制备

将中间体 7 (5.44 g, $0.012 \mathrm{~mol}) 、$ 、醇 $(60 \mathrm{~mL})$ 和浓氨 水 $(30 \mathrm{~mL})$ 加入反应瓶中, 室温摚拌反应 $12 \mathrm{~h}$, 反应结束 后, 减压蒸除溶剂, 残余物溶于二氯甲烷 $(60 \mathrm{~mL})$ 中, 用 饱和 $\mathrm{NaCl}$ 水溶液 $\left(30 \mathrm{~mL}\right.$ )洗涤, 有机层经无水 $\mathrm{Na}_{2} \mathrm{SO}_{4}$ 干燥, 减压蒸除溶剂, 残余物经硅胶柱层析纯化[洗脱 液: $V$ (石油醚) $: V($ 乙酸乙酯 $)=10 ： 1]$, 得 $4.2 \mathrm{~g}$ 淡黄色 油状物, 收率 $85.0 \%$. $[\alpha]_{\mathrm{D}}^{20}-15.8\left(c \quad 1.0, \mathrm{CH}_{3} \mathrm{OH}\right) ;{ }^{1} \mathrm{H}$ NMR $\left(\mathrm{CDCl}_{3}, 400 \mathrm{MHz}\right) \delta: 6.94 \sim 6.91(\mathrm{~m}, 3 \mathrm{H}, \mathrm{ArH}), 5.23$ (brs, $1 \mathrm{H}, \mathrm{NHCO}$ ), 3.89 (t, $J=4.4 \mathrm{~Hz}, 4 \mathrm{H}, 2 \times \mathrm{CH}_{2} \mathrm{O}$ ), $3.86 \sim 3.83\left(\mathrm{~m}, 2 \mathrm{H}, \mathrm{CHOH}+\mathrm{CH}_{2} \mathrm{NAr}\right), 3.60 \sim 3.57(\mathrm{~m}$, $1 \mathrm{H}, \mathrm{CH}_{2} \mathrm{NAr}$ ), $3.39 \sim 3.30\left(\mathrm{~m}, 1 \mathrm{H}, \mathrm{CH}_{2} \mathrm{NHCO}\right), 3.12(\mathrm{t}$, $\left.J=4.8 \mathrm{~Hz}, \quad 4 \mathrm{H}, \quad 2 \times \mathrm{CH}_{2} \mathrm{~N}\right), 3.08 \sim 3.04(\mathrm{~m}, 1 \mathrm{H}$, $\mathrm{CH}_{2} \mathrm{NHCO}$ ), 1.89 (s, 3H, $\left.\mathrm{CH}_{3}\right), 1.43\left(\mathrm{~s}, 9 \mathrm{H}, \mathrm{CH}_{3}\right) ;{ }^{13} \mathrm{C}$ NMR $\left(\mathrm{CDCl}_{3}, 100 \mathrm{MHz}\right) \delta: 172.8,156.8,154.5(\mathrm{~d}, J=$ $248.1 \mathrm{~Hz}), 139.9(\mathrm{~d}, J=8.3 \mathrm{~Hz}), 137.1(\mathrm{~d}, J=9.1 \mathrm{~Hz})$, 123.9 (d, $J=3.1 \mathrm{~Hz}), 118.9$ (d, $J=4.1 \mathrm{~Hz}), 115.7$ (d, $J=$ $21.5 \mathrm{~Hz}), 79.4,69.9,66.7,53.5,50.5,44.1,28.3,22.5$; HRMS (ESI) calcd for $\mathrm{C}_{20} \mathrm{H}_{31} \mathrm{FN}_{3} \mathrm{O}_{5}[\mathrm{M}+\mathrm{H}]^{+}$412.2248, found 412.2250 .

\section{$3.7 N$-[(2S)-3-氨基-2-羟基丙基]- $N$-[3-氟-4-(4-吗啉基)} 苯基]-乙酰胺二盐酸盐(3)的制备

反应瓶中加入中间体 $8(4.10 \mathrm{~g}, 0.01 \mathrm{~mol})$ 和无水乙 醚 $(20 \mathrm{~mL})$, 搅拌均匀后, 置冰浴中冷却至 $-10{ }^{\circ} \mathrm{C}$, 加 入饱和氯化氢乙醚溶液 $(20 \mathrm{~mL}), 0{ }^{\circ} \mathrm{C}$ 保温摚拌反应 $2 \mathrm{~h}$, 反应结束后，过滤析出的白色固体并用少量无水乙醚洗 涤, 所得滤饼置 $\mathrm{P}_{2} \mathrm{O}_{5}$ 干燥器中真空干燥, 得 $3.5 \mathrm{~g}$ 白色 粉末固体, 收率 $92.7 \%$, 纯度 $98.6 \%$ (HPLC 归一化法). m.p. $136.5 \sim 138.1{ }^{\circ} \mathrm{C} ;[\alpha]_{\mathrm{D}}^{20}+8.5\left(c 1.0, \mathrm{CH}_{3} \mathrm{OH}\right) ;{ }^{1} \mathrm{H}$ NMR $\left(\mathrm{D}_{2} \mathrm{O}, 400 \mathrm{MHz}\right) \delta: 7.52(\mathrm{t}, J=8.8 \mathrm{~Hz}, 1 \mathrm{H}, \mathrm{ArH})$, 7.39 (d, $J=12.8 \mathrm{~Hz}, 1 \mathrm{H}, \mathrm{ArH}), 7.34(\mathrm{~d}, J=8.8 \mathrm{~Hz}, 1 \mathrm{H}$, ArH), $4.17 \sim 4.14(\mathrm{~m}, 1 \mathrm{H}, \mathrm{CHOH}), 4.07$ (brs, 4H, $2 \times \mathrm{CH}_{2} \mathrm{O}$ ), 3.94 (dd, $J=4.4,14.4 \mathrm{~Hz}, 1 \mathrm{H}, \mathrm{CH}_{2} \mathrm{NAr}$ ), 3.77 (dd, $J=8.0,14.4 \mathrm{~Hz}, 1 \mathrm{H}, \mathrm{CH}_{2} \mathrm{NAr}$ ), 3.45 (brs, $4 \mathrm{H}$, $\left.2 \times \mathrm{CH}_{2} \mathrm{~N}\right), 3.22\left(\mathrm{~d}, J=12.8 \mathrm{~Hz}, 1 \mathrm{H}, \mathrm{CH}_{2} \mathrm{NH}_{2}\right), 2.99(\mathrm{dd}$, $\left.J=9.6,13.2 \mathrm{~Hz}, 1 \mathrm{H}, \mathrm{CH}_{2} \mathrm{NH}_{2}\right), 1.97\left(\mathrm{~s}, 1 \mathrm{H}, \mathrm{CH}_{3}\right) ;{ }^{13} \mathrm{C}$ NMR $\left(\mathrm{D}_{2} \mathrm{O}, 100 \mathrm{MHz}\right) \delta: 176.7,157.4(\mathrm{~d}, J=251.2 \mathrm{~Hz})$, 
146.7 (d, $J=9.4 \mathrm{~Hz}), 131.8,128.1,125.6,120.0$ (d, $J=$ $31.2 \mathrm{~Hz}$ ), 68.1, 66.9, 56.2, 54.9, 44.7, 24.4; HRMS (ESI) calcd for $\mathrm{C}_{15} \mathrm{H}_{23} \mathrm{FN}_{3} \mathrm{O}_{3}[\mathrm{M}+\mathrm{H}-2 \mathrm{HCl}]^{+} 312.1723$, found 312.1710 .

\section{$3.8 N$-[(2R)-3-[[3-氟-4-(4-吗啉基)苯基]氨基]-2-羟基} 丙基]-乙酰胺(2)的制备

将化合物 3 (2.0 g, $5.2 \mathrm{mmol})$ 、三乙胺 $(2.2 \mathrm{~mL}, 15.6$ $\mathrm{mmol}$ )和甲醇 $(20 \mathrm{~mL})$ 加入反应瓶中, 升温回流摚拌反应 $3 \mathrm{~h}$, 反应结束后, 减压蒸除溶剂, 残余物用二氯甲烷 $(25 \mathrm{~mL})$ 溶解, 依次用去离子水 $(20 \mathrm{~mL})$, 饱和 $\mathrm{NaCl}$ 水溶 液 $\left(20 \mathrm{~mL}\right.$ ) 洗涤, 有机层经无水 $\mathrm{Na}_{2} \mathrm{SO}_{4}$ 干燥, 减压蒸除 溶剂, 残余物经乙醇重结晶, 得 $1.81 \mathrm{~g}$ 白色固体, m.p. $124.6 \sim 125.5{ }^{\circ} \mathrm{C}$, 收率 $90.5 \%$.

\section{References}

[1] Zhang, H.; Du, K.; Shang, Z. P.; Deng, Y. Chin. J. Pharm. 2011, 42, 245 (in Chinese)

(张慧, 杜鲲, 桑志培, 邓勇, 中国医药工业杂志, 2011, 42, 245.)

[2] Clemett, D.; Markham, A. Drugs 2000, 59, 815.

[3] Hadden, C. E.; Bowman, P. B.; Duholke, W. H.; Guido, J. E.; Kaluzny, B. D.; Robins, R. H.; Russell, S. M.; Sims, S. M.; Thamann, T. J.; Martin, G. E. J. Heterocycl. Chem. 2000, 37, 1623.

[4] Wang, G. Anti-Infect. Agents Med. Chem. 2008, 7, 32.

[5] Barbachyn, M. R.; Ford, C. W. Angew. Chem., Int. Ed. 2003, 42, 2010.

[6] Wang, S. Q.; Deng, Y.; Chen, Z. X.; Zhang, H.; Sang, Z. P.; Mao, B. Y.; Xia, Z. J. WO 2012171248, 2012 [Chem. Abstr. 2012, 158, 77243].

[7] Babu, K. C.; Reddy, R. B.; Gangaiah, L.; Madhusudhan, G.; Mukkanti, K. Pharma Chem. 2011, 3, 219.

[8] Li, H. W.; Li, X. D.; Xie, W. B.; Zhang, L.; Wang, L. X. CN 102516191, 2012 [Chem. Abstr. 2012, 157, 165559].

(Zhao, C.) 